\title{
Effectiveness of plasma with low concentration of platelets combined with hyaluronic acid compared with platelet rich plasma in the conservative treatment of knee osteoarthritis. A retrospective study
}

\section{Michelangelo Palco}

Department of Biomedical and Dental Sciences and Morphological and Functional Images, Section of Orthopaedics and Traumatology, University of Messina, Italy

\section{Domenico Fenga}

Department of Biomedical and Dental sciences and of Morphological and Functional images, Section of Orthopaedics and Traumatology, University of Messina, Messina, Italy

\section{Paolo Rizzo}

Department of Biomedical and Dental sciences and of Morphological and Functional images, University of Messina, Messina, Italy

\section{Bruno Cavalieri}

Department of Biomedical and Dental sciences and of Morphological and Functional images, Section of Orthopaedics and Traumatology, University of Messina, Messina, Italy

\section{Demetrio Milardi}

Department of Biomedical and Dental sciances and gf Morphological Functional images, University of Messina, Messina, Italy

\section{Danilo Leonetti}

Department of Biomedical and Dental sciences and of Morphological and Functional images, Section of Orthopaedics and Traumatology, University of Messina, Messina,Italy

\section{Antongiulio Bruschetta}

Orthopaedic Institute of Southern Italy "Franco Scalabrino", Messina, Italy

\section{Angelo Alito}

U.O.C of Physical and Rehabilitation Medicine and Sport Medicine, Azienda Ospedaliera Universitaria "G. Martino" Messina, Italy

Giorgio Basile (D Giorgio.b30397@gmail.com )

Universita degli Studi di Messina Dipartimento di Scienze biomediche odontoiatriche e delle immagini morfologiche e funzionali https://orcid.org/0000-0002-9538-0717

Francesco Traina 
Department of Biomedical and Dental sciences, and of Morphological and Functional images, section of Orthopaedic and Traumatology, University of Messina, Messina, Italy

\section{Research article}

Keywords: L-PRP, KSS, VAS, Knee osteoarthritis

Posted Date: November 18th, 2020

DOl: https://doi.org/10.21203/rs.3.rs-107447/v1

License: (c) (1) This work is licensed under a Creative Commons Attribution 4.0 International License. Read Full License 


\section{Abstract}

Background: Knee osteoarthritis (KO) is one of the most common joint diseases, often determining knee pain and reduction of mobility with impact on the overall quality of life of the patients. Intra-articular injections of different formulations of platelet rich plasma (PRP) are an increasingly common nonsurgical treatment for KO. Recently, in order to combine the antinflammatory effect of platelet rich plasma and the viscosupplementation effect of hyaluronic acid, a formulation of plasma with relatively low concentration of platelets and very few leukocytes combined with hyaluronic acid (PRP+HA) has been proposed. The purpose of this study is to retrospectively compare the effectiveness of plasma with high concentration of platelets and leukocytes (L-PRP) with PRP+HA in patients with mild to moderate (Kellegren-Lawrence scale II-III grade) KO.

Materials and Methods: Among the 51 patients included, 28 have been treated with L-PRP, while 23 with $\mathrm{PRP}+\mathrm{HA}$. A retrospective evaluation at baseline (T0), after 3 months (T1) and 1 year (T2) has been performed. The outcome analyzed are the Knee Society Score(KSS), the Visuo Analogic Scale (VAS) (at T0,T1 and T2) and the Knee injury and Osteoarthritis Outcome Score(KOOS) (T0 and T2).

We evaluated change in mean scores within group among different time points using repeated measures ANOVA or paired t-test. Comparison between different groups of treatment has been performed using mixed ANOVA.

Results: The main finding is that, although the two treatments have been both effective, PRP+HA determined significantly better functional status, pain and mobility, measured with KSS.

Discussion: We observed a significant effect of the two formulations in exam in all the outcomes in analysis: both treatments were effective in improving pain, knee functional status and symptoms, and the benefits persisted after 3 and 12 months from the infiltrative therapy. Nevertheless, patients who received $\mathrm{PRP}+\mathrm{HA}$ injections presented higher knee mobility and better function (measured by KSS score) after one year.

Conclusions: This work compares clinical outcomes of L-PRP and PRP+HA treatment in patients with KO. Our results encourage the use of one of the two treatments in patients with mild to moderate KO. When possible, we suggest to the use of PRP+HA formulation, because it may determine better results in improving knee function and mobility.

\section{Introduction}

Knee osteoarthritis (KO) is a common degenerative joint disease, affecting approximately 250 million people worldwide; it often determines knee pain, which limits activity and impairs quality of life(1), determining a risk of mobility disability (defined as the need for help with walking or climbing stairs) greater than in any other medical condition in people $\geq 65$ years of age (2). KO can be treated with surgery or with non-surgical options. To date, there is no treatment able to prevent or arrest cartilage 
degeneration: non-surgical options, based on patients educations, rehabilitation and pharmacotherapy, can be administered to reduce swelling, pain and disability (3), their main aim being to modify the lifestyle, achieve pain control, delay disease progression and improve function. Among these treatments, intra-articular infiltrations with hyaluronic acid $(\mathrm{HA})$ or blood derivatives are employed to achieve pain control and restore knee function (3). Specifically, the presence of HA in the osteoarthritic joint is thought to have a beneficial effect: the introduction of eterologous HA in the arthritic joint is called viscosupplementation, and is considered crucial to restore the mechanical properties of synovial fluid, thus determining an analgesic, antinflammatory and condroprotective effect(4). The rationale behind the use of blood derivatives is the ability to provide bioactive molecules which could positively influence the joint environment, and should favour the regeneration of degenerating tissues: in particular, platelet rich plasma(PRP) has gained increasing attention due to the pool of growth factors stored in platelet agranules, that, according to recent studies, could take part in the regeneration of articular cartilage $(5,6)$ .Clinical effectiveness of HA injections, as well as safety, has been proven by several studies(7). In patients with mild-moderate KO, PRP administration shows comparable effectiveness to steroid/anaesthetic or HA (8). Furthermore, the use of PRP is appropriate to treat patients with severe KO not eligible for knee replacement due to pre-existing conditions or other personal reasons (8). The few available randomized controlled trials give overall support to PRP injections for knee OA treatment (6).

Several different formulations of PRP can be employed in the management of KO. Recently, in vitro studies compared the effects of plasma with relatively low concentration of platelets and very few leukocytes (PRP) with leucocyte and platelet rich plasma (L-PRP) on human chondrocytes (9). PRP attenuates inflammation, by reducing the production of inflammatory cytockines; conversely, it reduces the chondrocytic production of HA (9). Hence, in order to combine the antinflammatory effect of PRP and the viscosupplementation effect of HA, a new formulation (PRP $+\mathrm{HA}$ ) has been recently proposed (10).

Although several clinical reports have shown that the combination of PRP and HA could promote tissue regeneration, improve the mobility of patients, relieve pain and reduce the risk of infections, these studies are often based on a restricted number of patients. Furthermore, the molecular mechanism which should lead to tissue regeneration has been not completely described. Therefore, evidence supporting the effectiveness of this formulation is still considered limited and controversial $(10,11)$.

Additionally, to the best of our knowledge, there is no clinical evidence comparing pain perception and objective functional outcomes of PRP + HA with L-PRP treatment in patients with low to mild-moderate grade of $\mathrm{KO}$.

In this study, we review the outcomes of knee intra-articular injective therapy administered to outpatients treated in our structure between 2017 and 2018. Hence, the objective of this retrospective study is to report the effects of L-PRP and PRP + HA treatment on pain and knee function, and to compare the outcomes of the two treatments to determine wheter they are equivalent or not .

\section{Materials And Methods}




\section{Patient selection}

This retrospective study has been conducted at the Policlinic "Gaetano Martino" (Messina, Italy) and has been authorized by the local ethical committee. The study was designed as an observational study, aiming to find differences in the outcomes between patients treated with L-PRP and with PRP+HA.

We selectioned the participants among the outpatients suffering from KO treated in our facility between December 2017 and December 2018. Standard follow-up for patients treated with injective therapy lasts 1 year: therefore, follow-up of the last patient included in the study ended in the month of December 2019.

In all the patients, $\mathrm{KO}$ has been diagnosed and classificated by clinical evaluation and anteroposterior radiograph of the osteoarthritic knee. Radiographic appearance of the most involved knee was evaluated basing on the Kellegren-Lawrence staging system (KL)(12). According to $\mathrm{KL}, \mathrm{KO}$ can be doubtful (grade I); mild (grade II); moderate (grade III); severe (grade IV); the radiological evaluation of the patients has been performed by 2 experienced observers (M.P.-P.R.), with several years of work in the orthopedic field.

In case of doubt, the senior Author (F.T.) was always available to contribute to the evaluation. We considered patients not eligible to injective treatment if they presentied haematological, oncological diseases or low platelet count $\left(<150.000 / \mathrm{mm}^{3}\right)$. Due to the influence that the following conditions may have on the outcomes in exam, patients were excluded from the retrospective evaluation if they presented/reported : recent knee injuries or trauma, arthritis, moderate genu varus or valgus, bucket-like meniscal lesions, joint infections, bone necrosis, use of corticosteroids in the last 3 months, use of drugs or physical therapy (e.g. cryotherapy) for analgesic-anti-inflammatory purposes, history of spine or lower limb surgery. We furthermore excluded patients if they did not release informed consent, were not present to one follow-up visit, or (for any reason) underwent follow-up evaluation at an anticipated or delayed time point.

To evaluate the effects of the treatment in patients with mild to moderate disease we decided to exclude from our retrospective revision patients with severe or very low $\mathrm{KL}$ grade (grade I and IV).

We estimated sample size using G*Power software. We considered a minimal sample size of 44 subjects (22 per group): we adopted an a level=0.05, power $(1-\beta)=0.95$, and effect size of 0.25 (medium effect size) $(13,14)$.

We identified 51 patients suffering from knee osteoarthritis, treated with intra-articular injection of one of the two PRP formulations between December 2017 and December 2018, and meeting all the other selection criteria. Among the 51 patients, 23 have been treated with L-PRP and 28 with PRP+HA. From this point, we will consider the patients as part of two separate groups (L-PRP and PRP+HA). All the patients have received one infiltration in the most symptomatic knee every 15 days for a total of 3 injections in 30 days (on the $1^{\text {st }}, 15^{\text {th }}$ and $30^{\text {th }}$ day). 
Regardless of the treatment administered, physiotherapy and gradual muscle strengthening have been prescribed since the second infiltration of the cycle to all the patients.

\section{Treatment}

For each infiltration a single dose of $8 \mathrm{ml}$ of venous blood from the cubital vein is collected in a sterile test tube. Subsequently, we useRegenKit $\circledast$-THT-3 / RegenCell $®$ to obtain L-PRP and CellularMatrix A-CP-HA to obtain PRP + HA (REGEN LAB SA, En Budron B2,1052 Mont-sur-Lausanne,Switzerland).

L-PRP is obtained after centrifugation of venous blood for 9 minutes at $3400 \mathrm{rpm} / 1500 \mathrm{~g}$; PRP+HA instead, is centrifuged for 5 minutes at $3400 \mathrm{rpm} / 1500 \mathrm{~g}$. The volume obtained from each individual tube stands at $5 \mathrm{ml}$ (in the case of PRP+HA, $5 \mathrm{ml}$ equals to $3 \mathrm{ml}$ of PRP and $2 \mathrm{ml}$ of HA) (15).

\section{Outcome Measures}

To assess the efficacy of the treatment on knee function and pain, we evaluated patients using the Knee Society Score (KSS), the Knee injury and Osteoarthritis Outcome Score(KOOS), and the Visuo-Analogic Scale (VAS).

The KSS is based on therapist observation and comprises a knee score and a functional score: the first one evaluates aspects of mobility (range of motion, flexion contracture, extension lag, alignment, and stability in the anteroposterior and mediolateral plane), with a maximum score of 100 points. The functional score is based on walking (50 points), stair climbing (50 points), and on the possible use of walking aids (which can subtract up to 20 points). Scores of 80-100 are empirically considered excellent, 70-79 good, $60-69$ fair, and $<60$ poor (16) .

The KOOS is a self reported questionnaire rating five dimensions: pain, other symptoms, activities of daily living, sport and recreation function, and knee-related quality of life. Total scores from different subscales are translated to a total score ranging from 0 (extreme functional impairment) to 100 (no impairment) (17).

The Visual-Analogue Scale (VAS) has been in use for the measurement of intangible quantities (such as pain) since the 1920s. It consists of a line usually $100 \mathrm{~mm}$ in length, with anchor descriptors such as (in the pain context) "no pain" and "worst pain imaginable". The patient marks the line to indicate the pain perception: the outcome is represented by the distance from the left endpoint to the mark, measured in $\mathrm{mm}(18)$.

Each patient has been evaluated with VAS and KSS before treatment (baseline evaluation: T0), 3 months (T1) and 12 months (T2) after treatment. KOOS was administered at T0 and T2. The administration the therapy, has been performed in outpatient regimen, as well as the clincal outcomes, collected in the context of the general follow-up after injective therapy.

\section{Statistical analysis}


We analyzed the differences between the two groups for gender, $\mathrm{KL}$ grade $\left(\chi^{2}\right.$ test) and mean age (t-test). At baseline, the two groups mean scores of outcome variables (KOOS, KSS and VAS) were compared using independent-samples t-test.

In order to evaluate the difference in KOOS score within groups before and after treatment, we compared mean KOOS scores (T0-T2) using paired t-test.

We used repeated measures ANOVA with post-hoc pairwise analysis (Bonferroni) to compare VAS and KSS scores within groups at the three time points of the study $(\mathrm{T} 0, \mathrm{~T} 1, \mathrm{~T} 2)$.

For each of the three outcome variables considered (KSS, KOOS, VAS), we analyzed the mean scores using a mixed ANOVA model with time as within subject variable and treatment type as between subject variable, to determine if there was a significant main effect of the treatment type on the knee functional and pain outcomes.

Bonferroni correction for multiple comparisons was adopted: thus the significance level was set at $p=0.008(p=0.05 / 6)$.

All statistical analysis have been performed with SPSS version 25.0 (IBM Corp, Armonk, NY).

\section{Results}

All patients included in the statistical analyisis received the treatment without any notice of harmful/unespected effects.

At the moment of the injection, out of 51 patients, 18 (35.29\%) were suffering from a II KL grade KO, while 33 patients $(64.71 \%)$ from a III grade. Among the 51 patients, 27 were females $(52.94 \%)$ and 24 males (47.06\%).

Groups did not show differences for gender and $\mathrm{KL}$ grade, but patients treated with PRP+HA had an higher mean age $(p=0.002)$. All the baseline characteristics have been summarised in Table 1.

Due to the mean age difference between groups, we proceeded adopting Pearson correlation coefficient to determine if age was correlated with KSS, KOOS or VAS: however, this analysis highlighted no correlation with any of the quantitative measures in exam.

\section{Effect of L-PRP and PRP+HA}

Both groups showed significant improvements in VAS, KSS and KOOS at the 1-year post-treatment assessment: outcome scores(mean \pm standard deviations), significance and effect sizes are summarized in Table 2. 
In L-PRP group, post-hoc Bonferroni pairwise comparison did not show a significant difference between T1 and T2 KSS scores; in PRP+HA group instead, values at T2 were significantly better than at T1 $(p=0.008)$.

\section{L-PRP vs PRP+HA}

For KSS, comparison between L-PRP and PRP+HA highlighted a significant interaction between time and treament $\left(F(2,98)=14.887, p<0.001, \eta^{2}=0.233\right)$. In particular, we highlighted a significant simple main effect of treatment both at $\mathrm{T} 1\left(\mathrm{~F}(1,49)=12.401, \mathrm{p}=0.001, \eta^{2}=0.202\right)$ and $\mathrm{T} 2(\mathrm{~F}(1,49)=11.015, \mathrm{p}=0.002$, $\eta^{2}=0.184$ ), with PRP+HA leading to higher scores (mean difference:T1=5.592; T2=7.839). Figure 1 shows the difference of KSS scores for the two different treatment groups. In KOOS and VAS scores instead, we did not find any significant difference between groups (Figure 1).

\section{Discussion}

The present study reported the comparison of L-PRP with PRP + HA infiltrative treatment in KO.

Our results show that both treatments have been effective to obtain a reduction of pain and an improvement in mobility of the osteoarthritic knee, as showed by significant change of the examined outcomes. In addition, patients in KL II-III stages of KO treated either with L-PRP or PRP + HA showed satisfactory results, but those receiving PRP + HA injections presented higher knee mobility and better function (measured by KSS score) after 1 year.

As previously mentioned, these two treatment have been already compared in vitro on human articular chondrocytes (9). Cavallo and colleagues reported how L-PRP stimulates chondrocytic secrection of HA, which determines a positive effect due to its viscoelastic, analgesic, anti-inflammatory and chondroprotective properties; in the other hand, they suggested that PRP could reduce inflammatory cytokines production (9). Most recently, clinical studies have shed new light on the different properties of $\mathrm{PRP}, \mathrm{HA}$, and PRP + HA in patients with $\mathrm{KO}$. Examining pain perception and knee function in patients treated with either PRP or HA, Di Martino et al. observed in both groups a significant improvement in symptoms, function and activity which remained stable over time up to 24 months. The authors observed a pain relieving effect up to 9 months for HA and 12 months for PRP, but these results did not reach significance. Furthermore, a significantly lower rate of reintervention at 24 months was reported in the PRP group (19). Our results are consistent with the aforementioned study, by showing a 1-year significant pain reduction with both the PRP formulations employed.

In addition, it is worthy to note that in our work pain relief was still present at the last follow-up. Not only VAS, but also KOOS and KSS showed persistent improvement: specifically, for KSS, pairwise comparison highlighted that L-PRP group significantly improved from baseline to T1 (3 months), but remained stable from T1 to T2 (1 year). Otherwise, PRP + HA group showed significant improvement up to the 1-year follow-up (T2): this result suggests that while L-PRP effect reached a plateau, remaining stable after T1 
(from 3 months to 1 year), PRP + HA effect on the injured joint sustainedly grew over time (still improving after 1 year).

Also Lana et al. compared the effects of the administration of PRP + HA, PRP and HA in patients with mild-moderate $\mathrm{KO}(20)$. In the cited study, the PRP-treated group had a significant reduction in pain (VAS) after $1,3,6$, and 12 months and a greater improvement in physical function after 12 months; indeed, PRP $+\mathrm{HA}$ showed better outcomes in pain and functional limitation when compared to HA alone at 1 year post treatment and significantly increased physical function at 1 and up to 3 months when compared to PRP alone. The authors described the use of autologous PRP as an effective treatment of mild to moderate knee osteoarthritis, and the use of PRP + HA as a treatment of choice, due to better results after 1 and 3 months (20). Although we substantially agree to consider both PRP and PRP HA effective in KO and to prefer the use of PRP + HA due to patient's higher functional improvement,we have to point out that our comparison of L-PRP and PRP + HA yielded slightly different results, highlighting a significant improvement in functional (KSS) scores not only after 3 months, but also after 1 year. It is worthy to note that while Lana et al. used only patient-reported outcomes, in this work we administered KSS (depending on objective scores) which has been the only outcome measure to differ between groups. The use of different measures could have determined the discrepancy between the results: even the authors suggested that the use of only self-reported questionnaires could limit the objectivity of the results (20). Also in our study, for instance, no significant effect of treatment was observed in patient-reported measures.

An important element, which may possibly affect the outcome of injective treatment, is the stage of disease of the patients: most of the studies regarding conservative therapy in $\mathrm{KO}$ include mainly patients in early stages, whereas grade III and IV are less represented, while Meheux's and colleagues reported that the worst results are achieved for K-L grade III to IV (21). The aim of the current study was to compare the two PRP formulations only in patients with KL II-III, but could be interesting and relevant to describe the effects of these treatment specifically in patients with severe KO (IV KL grade). Our clinical experience suggests that both L-PRP and PRP + HA could induce improved function, mobility and pain relief also in patients with severe KO; nevertheless further evidence is needed to understand wether, and to what extent, different PRP formulations could actually improve their clinical conditions.

The reduced sample size is a limitation of this study, as well as the significant difference in the mean age of the two groups in exam: however, as we reported previously, we did not find any correlation between age and any of the outcomes in exam. Another limitation to this study is the absence of follow-up after 1year: at this time point, the benefits of treatment were still observable, so we were not able to determine how much they persisted, or if the outcomes lasted longer in one of the two groups compared with the other.

\section{Conclusions}


To sum up, we observed a significant effect of the two formulations in exam in all the outcomes in analysis: both treatments were effective in improving pain, knee functional status and symptoms, and the benefits persisted after 3 and 12 months from the infiltrative therapy.

The group treated by intra-articular injection of PRP enriched with HA showed an higher improvement in functional outcome and knee mobility, but not in pain and self reported level of activity.

In our point of view, we encourage the use of PRP injection as a simple, safe and minimally invasive treatment approach. Specifically, when possible, we suggest to prefer the use of PRP in combination with HA. Further studies could better describe the use and the efficacy of this biological treatment, comparing it to other non surgical options or studiyng its effects in severe OA.

\section{Abbreviations}

KO: Knee Osteoarthritis; PRP = Platelet Rich Plasma; L-PRP = leucocytes and platelets rich plasma; PRP = pure platelet rich plasma; $\mathrm{HA}=$ hyaluronic acid; $\mathrm{KL}=$ Kellegren Lawrence scale; $\mathrm{KOOS}=$ Knee Injury and Osteoarthritis Outcome Score; KSS = Knee Society Score; VAS = Visual Analogue Scale; IKDC = International Knee Documentation Committee;WOMAC = The Western Ontario and McMaster Universities Arthritis Index.

\section{Declarations}

\section{Ethics approval and consent to participate:}

This work was approved by the Ethical Committee of Polyclinic "Gaetano Martino" (Messina). All participants released informed consent during outpatient visits at our departement before the inclusion in the work. Registration number: Comitato Etico di Messina, $n^{\circ} 0014580$

Consent to publication: not applicable.

Competing interest: All authors declare that they have no competing interests in the publication of this work.

Funding: no funding was received for this work.

Availability of data and material: The datasets used and/or analysed during the current study are available from the corresponding author on reasonable request.

Authors'contribution: MP, DF, PR, BC proposed the topic of the study, and contributed to patient's selection, data collection and statistical analysis. AB, DM, DL, and GB wrote the article and supervised data selection. GB performed statistical analysis. FT contributed to patients selection, and coordinated the authors. All authors supervised the final version of the work. 


\section{References}

1. Hunter DJ. Viscosupplementation for osteoarthritis of the knee. N Engl J Med. 2015;

2. Murray CJL, Vos T, Lozano R, Naghavi M, Flaxman AD, Michaud C, et al. Disability-adjusted life years (DALYs) for 291 diseases and injuries in 21 regions, 1990-2010: A systematic analysis for the Global Burden of Disease Study 2010. Lancet. 2012;

3. Hussain SM, Neilly DW, Baliga S, Patil S, Meek RMD. Knee osteoarthritis: A review of management options. Scottish Medical Journal. 2016.

4. Shen L, Yuan T, Chen S, Xie X, Zhang C. The temporal effect of platelet-rich plasma on pain and physical function in the treatment of knee osteoarthritis: Systematic review and meta-analysis of randomized controlled trials. J Orthop Surg Res. 2017;

5. Filardo G, Madry H, Jelic M, Roffi A, Cucchiarini M, Kon E. Mesenchymal stem cells for the treatment of cartilage lesions: From preclinical findings to clinical application in orthopaedics. Knee Surgery, Sports Traumatology, Arthroscopy. 2013.

6. Görmeli G, Görmeli CA, Ataoglu B, Çolak C, Aslantürk O, Ertem K. Multiple PRP injections are more effective than single injections and hyaluronic acid in knees with early osteoarthritis: a randomized, double-blind, placebo-controlled trial. Knee Surgery, Sport Traumatol Arthrosc. 2017;

7. Xing D, Wang B, Liu Q, Ke Y, Xu Y, Li Z, et al. Intra-articular Hyaluronic Acid in Treating Knee Osteoarthritis: A PRISMA-Compliant Systematic Review of Overlapping Meta-analysis. Sci Rep. 2016;

8. Cerza F, Carnì S, Carcangiu A, Di Vavo I, Schiavilla V, Pecora A, et al. Comparison between hyaluronic acid and platelet-rich plasma, intra-articular infiltration in the treatment of gonarthrosis. Am J Sports Med. 2012;

9. Cavallo C, Filardo G, Mariani E, Kon E, Marcacci M, Pereira Ruiz MT, et al. Comparison of platelet-rich plasma formulations for cartilage healing: An in vitro study. J Bone Jt Surg - Ser A. 2014;

10. lio K, Furukawa KI, Tsuda E, Yamamoto Y, Maeda S, Naraoka T, et al. Hyaluronic acid induces the release of growth factors from platelet-rich plasma. Asia-Pacific J Sport Med Arthrosc Rehabil Technol. 2016;

11. Shimojo AAM, Duarte A da SS, Lana JFSD, Luzo ângela CM, Fernandes AR, Sanchez-Lopez E, et al. Association of platelet-rich plasma and auto-crosslinked hyaluronic acid microparticles: Approach for orthopedic application. Polymers (Basel). 2019;

12. KELLGREN JH, LAWRENCE JS. Radiological assessment of osteo-arthrosis. Ann Rheum Dis. 1957;

13. Cohen J. 2.2. The Effect Size Index: d. In: Statistical Power Analysis for the Behavioral Sciences. 1988.

14. Faul F, Erdfelder E, Lang AG, Buchner A. G*Power 3: A flexible statistical power analysis program for the social, behavioral, and biomedical sciences. In: Behavior Research Methods. 2007.

15. Alves R, Grimalt R. A Review of Platelet-Rich Plasma: History, Biology, Mechanism of Action, and Classification. Skin Appendage Disorders. 2018. 
16. Insall JN, Dorr LD, Scott RD, Scott WN. Rationale of The Knee Society clinical rating system. In: Clinical Orthopaedics and Related Research. 1989.

17. Roos EM, Roos HP, Lohmander LS, Ekdahl C, Beynnon BD. Knee Injury and Osteoarthritis Outcome Score (KOOS) - Development of a self-administered outcome measure. J Orthop Sports Phys Ther. 1998;

18. Nahler G, Nahler G. visual analogue scale (VAS). In: Dictionary of Pharmaceutical Medicine. 2009.

19. Di Martino A, Di Matteo B, Papio T, Tentoni F, Selleri F, Cenacchi A, et al. Platelet-Rich Plasma Versus Hyaluronic Acid Injections for the Treatment of Knee Osteoarthritis: Results at 5 Years of a DoubleBlind, Randomized Controlled Trial. Am J Sports Med. 2019;

20. Lana JFSD, Weglein A, Sampson SE, Vicente EF, Huber SC, Souza C V., et al. Randomized controlled trial comparing hyaluronic acid, platelet-rich plasma and the combination of both in the treatment of mild and moderate osteoarthritis of the knee. J Stem Cells Regen Med. 2016;

21. Meheux CJ, McCulloch PC, Lintner DM, Varner KE, Harris JD. Efficacy of Intra-articular Platelet-Rich Plasma Injections in Knee Osteoarthritis: A Systematic Review. Arthroscopy - Journal of Arthroscopic and Related Surgery. 2016.

\section{Tables}

Table 1: Demographics of the patients in study. Age is expressed as mean \pm standard deviation (M $\pm S D)$.

\begin{tabular}{|llll|}
\hline Treatment group & & PRP & PRP+HA \\
\hline Age & & $54,04 \pm 10,4$ & $62,71 \pm 7,88$ \\
\hline Sex & M/F & $12 / 11$ & $12 / 16$ \\
\hline K-L grade & II/III & $10 / 13$ & $8 / 20$ \\
\hline
\end{tabular}

Table 2: VAS, KSS and KOOS scores of the two groups. Repeated measures ANOVA/t-test showed a significant improvement in all the variables in exam. Scores are expressed as mean \pm standard deviation (M $\pm S D)$. 


\begin{tabular}{|llllll|}
\hline & T0 & T1 & T2 & $\begin{array}{l}\text { p-value; } \\
\text { (within subjects) }\end{array}$ & \\
\hline VAS (L-PRP) & $75.65 \pm 8.16$ & $43.70 \pm 14.48$ & $22.61 \pm 16.01$ & $p<0.001$ & $\eta^{2}=0.903$ \\
\hline VAS (PRP+HA) & $75.89 \pm 8.5$ & $38.75 \pm 10.68$ & $25.18 \pm 15.48$ & $p<0.001$ & $\eta^{2}=0.913$ \\
\hline KSS (L-PRP) & $71.13 \pm 3.21$ & $79.60 \pm 4.72$ & $81.00 \pm 6.64$ & $p<0.001$ & $\eta^{2}=0.833$ \\
\hline KSS (PRP+HA) & $70.91 \pm 4.65$ & $85.20 \pm 6.29$ & $88.83 \pm 9.58$ & $p<0.001$ & $\eta^{2}=0.820$ \\
\hline KOOS (L-PRP) & $64.53 \pm 22.56$ & $/ /$ & $83.17 \pm 14.28$ & $p<0.001$ & $\eta^{2}=0.368$ \\
\hline KOOS (PRP+HA) & $56.17 \pm 21.80$ & $/ /$ & $79.34 \pm 17.74$ & $p<0.001$ & $\eta^{2}=0.343$ \\
\hline
\end{tabular}

\section{Figures}
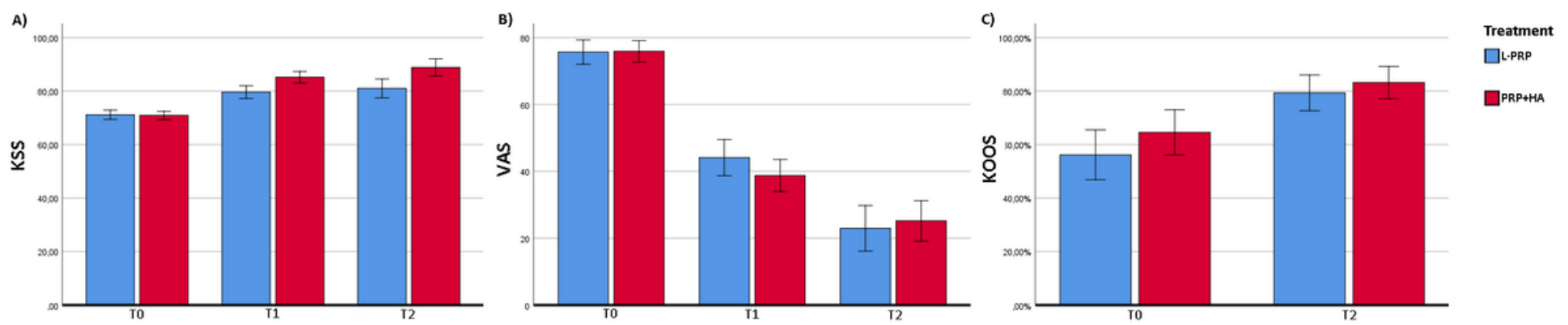

Figure 1

Histograms showing marginal means of KSS (A), VAS (B) and KOOS (C) at the different time points of the study. Error bars show the $95 \%$ confidence interval. The only significant difference concerned KSS score, which was better in patients treated with PRP+HA (A).
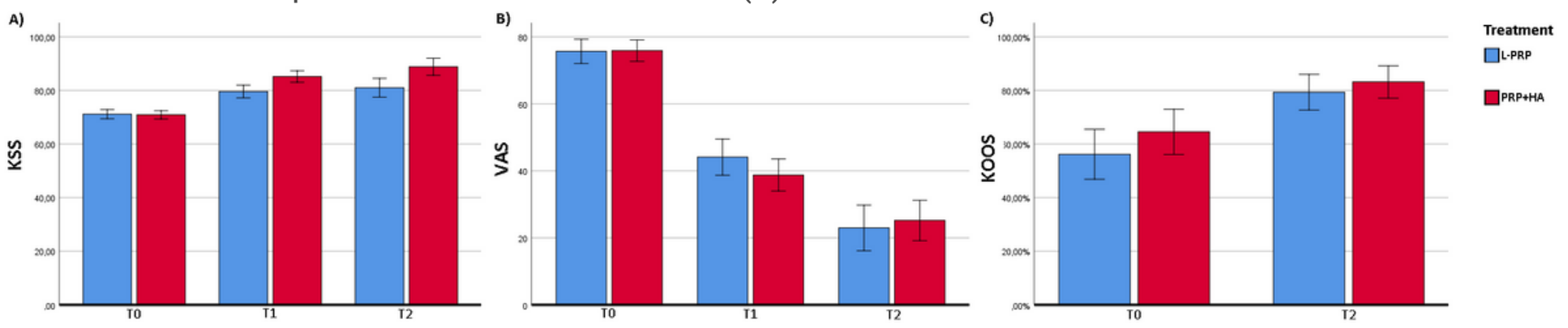

Figure 1

Histograms showing marginal means of KSS (A), VAS (B) and KOOS (C) at the different time points of the study. Error bars show the $95 \%$ confidence interval. The only significant difference concerned KSS score, which was better in patients treated with PRP+HA (A). 